\title{
Lateral lumbar interbody fusion in the elderly: a 10-year experience
}

\author{
Presented at the 2018 AANS/CNS Joint Section on Disorders of the Spine and Peripheral Nerves \\ Nitin Agarwal, MD, Andrew Faramand, MD, MSc, Nima Alan, MD, Zachary J. Tempel, MD, \\ D. Kojo Hamilton, MD, David O. Okonkwo, MD, PhD, and Adam S. Kanter, MD \\ Department of Neurological Surgery, University of Pittsburgh Medical Center, Pittsburgh, Pennsylvania
}

\begin{abstract}
OBJECTIVE Elderly patients, often presenting with multiple medical comorbidities, are touted to be at an increased risk of peri- and postoperative complications following spine surgery. Various minimally invasive surgical techniques have been developed and employed to treat an array of spinal conditions while minimizing complications. Lateral lumbar interbody fusion (LLIF) is one such approach. The authors describe clinical outcomes in patients over the age of 70 years following stand-alone LLIF.
\end{abstract}

METHODS A retrospective query of a prospectively maintained database was performed for patients over the age of 70 years who underwent stand-alone LLIF. Patients with posterior segmental fixation and/or fusion were excluded. The preoperative and postoperative values for the Oswestry Disability Index (ODI) were analyzed to compare outcomes after intervention. Femoral neck t-scores were acquired from bone density scans and correlated with the incidence of graft subsidence.

RESULTS Among the study cohort of 55 patients, the median age at the time of surgery was 74 years (range 70-87 years). Seventeen patients had at least 3 medical comorbidities at surgery. Twenty-three patients underwent a 1-level, 14 a 2-level, and 18 patients a 3-level or greater stand-alone lateral fusion. The median estimated blood loss was $25 \mathrm{ml}$ (range 5-280 ml). No statistically significant relationship was detected between volume of blood loss and the number of operative levels. The median length of hospital stay was 2 days (range 1-4 days). No statistically significant relationship was observed between the length of hospital stay and age at the time of surgery. There was one intraoperative death secondary to cardiac arrest, with a mortality rate of $1.8 \%$. One patient developed a transient femoral nerve injury. Five patients with symptomatic graft subsidence subsequently underwent posterior instrumentation. A lower femoral neck t-score $<-1.0$ correlated with a higher incidence of graft subsidence $(p=0.006)$. The mean ODI score 1 year postoperatively of 31.1 was significantly ( $p=0.003$ ) less than the mean preoperative ODI score of 46.2 .

CONCLUSIONS Stand-alone LLIF can be safely and effectively performed in the elderly population. Careful evaluation of preoperative bone density parameters should be employed to minimize risk of subsidence and need for additional surgery. Despite an association with increased comorbidities, age alone should not be a deterrent when considering stand-alone LLIF in the elderly population.

https://thejns.org/doi/abs/10.3171/2018.3.SPINE171147

KEYWORDS lateral lumbar interbody fusion; elderly; outcomes; complications

$\mathrm{W}$ ITH the advances in medical care, it is expected that $53 \%$ of the United States population will be above the age of 65 by $2020 .^{7}$ According to the World Health Organization, musculoskeletal conditions are the most disabling in people over the age of 70 , and back pain is the most common complaint. ${ }^{29}$ It is estimated that the prevalence of back pain among patients over the age of 65 is about $56 \% .{ }^{29}$ Lumbar fusion has been utilized for the treatment of a variety of spinal pathologies including degenerative diseases causing low-back pain. ${ }^{3,5,16}$ How-

ABBREVIATIONS ALIF = anterior lumbar interbody fusion; EBL = estimated blood loss; LLIF = lateral lumbar interbody fusion; ODI = Oswestry Disability Index; PLIF = posterior lumbar interbody fusion; TLIF = transforaminal lumbar interbody fusion.

SUBMITTED October 24, 2017. ACCEPTED March 14, 2018.

INCLUDE WHEN CITING Published online July 27, 2018; DOI: 10.3171/2018.3.SPINE171147. 
ever, elderly patients, often presenting with multiple medical comorbidities, are touted to be at an increased risk of peri- and postoperative complications.

Minimally invasive spine surgery was first presented as an alternative to open surgery in $1991 .{ }^{22}$ Lateral lumbar interbody fusion (LLIF) is a surgical technique that entails gaining access to the lumbar spine through a lateral approach by passing through the retroperitoneal fat and the psoas major muscle. ${ }^{23}$ The main advantages of this approach include decreased intraoperative blood loss, lower intraoperative narcotic requirements, a shorter hospital stay, and lower risk of intraoperative injury compared to open surgery. ${ }^{8,15,20}$

The clinical outcomes in elderly patients undergoing stand-alone LLIF are not well established. As such, we report the clinical outcomes and complications in patients over the age of 70 who underwent stand-alone LLIF for the management of degenerative spinal pathology.

\section{Methods}

A total of 470 patients underwent LLIF in the period between 2007 and 2016. Ninety-seven patients were 70 years of age or older at the time of surgery. Patients who underwent LLIF combined with posterior supplementary fixation or fusion were excluded. A retrospective analysis of this prospectively maintained database was performed on the study cohort consisting of 55 patients; inclusion criteria were as follows: age over 70 years at time of intervention with a minimally invasive stand-alone LLIF. All procedures were performed at a single institution. The LLIF procedure was performed as previously described. ${ }^{13}$ Patients underwent DEXA scans preoperatively to determine bone density. Routine postoperative radiographs and $\mathrm{CT}$ scans of the abdomen and pelvis were obtained to assess for radiographic endplate breach as well as occult injury to peritoneal or retroperitoneal structures.

Electronic medical records were reviewed for patient demographics, procedures, clinical outcomes, and complications. Functional outcomes were reported using Oswestry Disability Index (ODI) scores preoperatively and at 6 weeks, 3 months, 6 months, and 1 year following surgery. Femoral neck t-scores were acquired from bone density scans. These were utilized to establish a correlation with graft subsidence.

Statistical analysis was performed with SPSS Statistics 24 (IBM). Differences in clinical outcomes scores from preoperative to 1 -year postoperative time-points were determined using t-tests. Univariate analysis was done to study the correlation between the numbers of levels operated on and the length of hospital stay and risk of postoperative complications. Additionally, univariate analysis was done to study the correlation between age and length of stay. A p value $<0.05$ was set for statistical significance.

\section{Results}

Among the study cohort of 55 patients, 36 patients were women and 19 were men. The median age at the time of surgical intervention was 74 years (range 70-87 years). Seventeen patients had at least 3 medical comorbidities at
TABLE 1. Patient demographics

\begin{tabular}{lc}
\hline \multicolumn{1}{c}{ Characteristic } & Value \\
\hline Median age (range), yrs & $74(70-87)$ \\
\hline Sex & 36 \\
\hline Female & 19 \\
\hline Male & \\
Medical comorbidities & 37 \\
Hypertension & 13 \\
Coronary artery disease & 15 \\
Hyperlipidemia & 14 \\
Cancer & 14 \\
Diabetes mellitus & 3 \\
Venous thromboembolism & \\
No. of levels fused & 23 \\
1 & 14 \\
2 & 18 \\
3 or more &
\end{tabular}

the time of surgery. The most common medical comorbidities are presented in Table 1. The mean follow-up was 15 months (range 1-48 months). Twenty-three patients underwent a 1-level fusion, 14 a 2-level fusion, and 18 patients a 3-level or higher fusion. The median estimated blood loss (EBL) was $25 \mathrm{ml}$ (range 5-280 ml). None of the patients required intraoperative or postoperative blood transfusion. No statistically significant relationship was revealed between volume of blood loss and the number of operative levels. The median length of hospital stay was 2 days (range 1-4 days). No statistically significant relation was found between the length of hospital stay and age at the time of surgery.

\section{Clinical Outcomes}

The mean preoperative ODI score was 46.2 (range $8-80$ ). The mean postoperative ODI score at 6 months was 24 (range, $0-78$ ), and the mean ODI score 1 year postoperatively was 31.1 (range $0-68$ ). ODI scores documented 1 year postoperatively were $33 \%$ lower than the preoperative ODI scores ( $\mathrm{p}=0.003)$ (Fig. 1).

\section{Complications}

There was one intraoperative death secondary to cardiac arrest as a result of presumed myocardial infarction or pulmonary embolism, yielding an overall mortality rate of $1.8 \%$. The deceased patient had a history of coronary artery disease and was status post stenting, atrial fibrillation status post pacemaker, and tobacco use. One patient developed a transient femoral nerve injury. There were no visceral or vascular injuries in this series. Five patients with radiographic graft subsidence subsequently required posterior instrumentation and fusion. A summary of complications is presented in Table 2. DEXA scan reports were available in 23 patients. A lower femoral neck t-score $<-1.0$ correlated with a higher incidence of graft subsidence $(p=0.006)$. A greater number of levels operated on did not correlate with a higher risk of developing complications. The number of medical comorbidities was not associated with a higher risk of complications. 
60

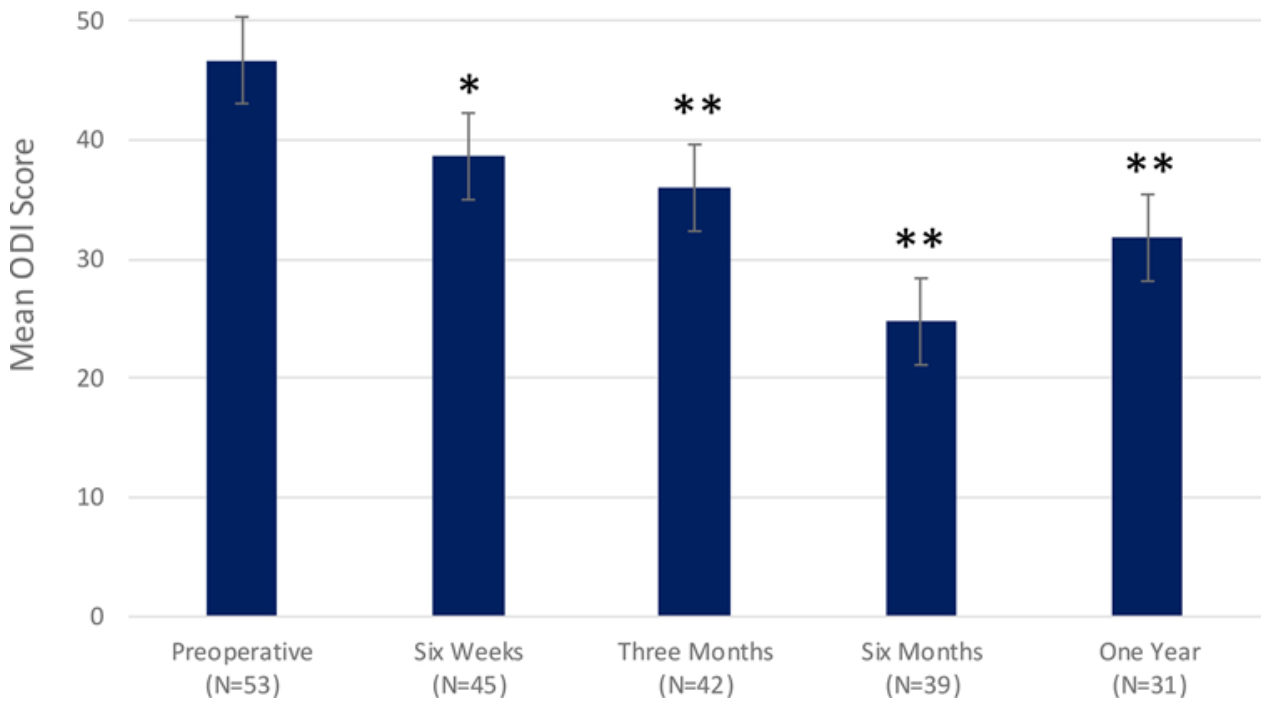

FIG. 1. Mean ODI score preoperatively and at 6 weeks, 3 months, 6 months, and 1 year postoperatively. $N$ represents the number of patients at each follow-up time interval. ${ }^{*} p<0.05 .{ }^{* *} p<0.005$. Figure is available in color online only.

\section{Discussion}

\section{Patient Characteristics}

The goal of minimally invasive spine surgery is to provide effective symptomatic relief with minimal tissue damage and minimal postoperative complications. To that end, elderly patients, often presenting with multiple comorbidities, benefit from lower intraoperative blood loss, faster wound healing, and early mobilization. In this cohort, we report a median hospital stay of 2 days. This is shorter than the length of stay reported in the literature for patients undergoing posterior lumbar interbody fusion (PLIF), transforaminal lumbar interbody fusion (TLIF), and anterior lumbar interbody fusion (ALIF) with an average range of 3-6 days. ${ }^{11,12,25}$ Karikari et al. reported a mean length of stay of 3.5 days in patients over the age of 70 who underwent stand-alone LLIF. ${ }^{14}$ We report a median EBL of $25 \mathrm{ml}$. This is in contrast to a reported mean EBL of $1147 \mathrm{ml}$ and $226 \mathrm{ml}$ in PLIF and TLIF, respectively. ${ }^{12}$ Karikari et al. also reported a mean EBL of $46 \mathrm{ml}$ in patients undergoing stand-alone 1- to 2-level LLIF and $175-\mathrm{ml}$ blood loss in more than 2-level LLIF.14 Avila et al. reviewed the outcomes of elderly patients undergoing minimally invasive spine surgery using various surgical approaches. ${ }^{1}$ They reported a mean blood loss of $43 \mathrm{ml}$ and a length of hospital stay of 1.7 days. It is worth noting, however, that all patients in their cohort underwent single-level intervention and that the majority of their patients underwent intralaminar decompression and fusion. In this cohort, no statistically significant correlation was observed between the number of levels and the EBL. Rodgers et al. reported outcomes in patients over the age of 80 years who underwent either open PLIF or minimally invasive LLIF. They found that LLIF patients had lower mortality rates, lower complication rates, less intraoperative blood loss, and significantly shorter hospital stays. ${ }^{27}$

\section{Functional Outcomes}

Our study showed a statistically significant improvement in ODI score in as early as 6 weeks postoperatively, as well as on long-term follow-up. Based on ODI score interpretation, patients in this cohort were classified as either incapacitated or severely disabled before surgery. We observed a $33 \%$ improvement the 1-year postoperative ODI score. Postoperative ODI score interpretation suggests that patients had substantial degrees of improvement in their disability. Improvement in the functional score reported in this series is comparable to that of prior results of interbody fusion cited in the literature. Ozgur et al. reported a 39\% improvement in ODI score following an anterolateral transpsoas approach for fusion. ${ }^{23}$ Malham et al. reported a $44 \%$ improvement in ODI score in a cohort of 40 patients (mean age 65 years) who underwent standalone LLIF. ${ }^{19}$ In their study of TLIF outcomes, Nikhil et al. found that both functional outcomes and complication rates were comparable between the different age groups included in their cohort. ${ }^{21}$

\section{Complications}

The morbidity rates associated with spinal procedures

TABLE 2. Complications observed in patients who underwent stand-alone LLIF

\begin{tabular}{lc}
\hline Complication & No. $(\%)$ \\
\hline Mortality & $1(1.8)$ \\
\hline Subsidence & $5(9)$ \\
\hline Neurological & $1(1.8)$ \\
Hollow viscus & 0 \\
Vascular & 0 \\
\hline
\end{tabular}


in the elderly documented in the literature range between $25 \%$ and $80 \%$, and the mortality rate is $2 \%$. $^{2,4,9,14,26} \mathrm{Ob}$ served graft subsidence requiring surgical revision was $5 / 55(9 \%)$ in the present study. This is lower than the complication rates reported by Knight et al. and Grimm et al., who showed complication rates of $23 \%$ and $22.5 \%$, respectively. ${ }^{10,17}$ Karikari et al. reported a $32 \%$ complication rate. In their report, 4 patients who underwent stand-alone LLIF developed graft subsidence and required further surgery. ${ }^{14}$ Phan et al. reported on outcomes following ALIF in patients 65 years of age or older. ${ }^{24}$ They found that patients in this age group have a higher chance of developing graft subsidence compared to younger patients. Graft subsidence had a statistically significant correlation with femoral neck t-scores $<-1.0$ on DEXA scans. This finding is in line with the findings of Tempel et al., who reported a higher degree of graft subsidence in patients with t-scores $<-1.0$. This suggests that patients with poor bone density may benefit from supplementation of LLIF with posterior instrumentation. ${ }^{30}$ Other variables reportedly found to increase the risk of graft subsidence include over-distraction of the disc space, length of construct, cage width, endplate violation, and lateral plate fixation. ${ }^{6}, 18,28,30,31$ The clinical sequelae of graft subsidence may be more profound in patients who undergo stand-alone LLIF than those who undergo pedicle screw fixation. As such, before stand-alone LLIF, patients are typically screened for subsidence risk factors including age and bone mineral density. Patients with evidence of osteopenia or osteoporosis on DEXA scanning are typically offered LLIF with supplemental pedicle screw fixation in to reduce the risk of subsidence.

Carreon et al. found that the prevalence of complications is related to the age of the patient at the time of surgery, the number of levels fused, and the EBL. ${ }^{2}$ In this series, the number of levels and EBL did not correlate with the risk of complications. In addition, we did not find a statistically significant relationship between the number of medical comorbidities and the risk of developing a postoperative complications. This is in contrast to Karikari et al., who reported a higher rate of postoperative complications in patients with a higher number of medical comorbidities. ${ }^{14}$ Overall, complications rates for this study cohort were comparable or lower than those reported in the literature.

\section{Conclusions}

Lateral lumbar interbody fusion can be performed in the elderly with a relatively low rate of complications. This approach is associated with a high rate of symptomatic and functional improvement on short- and long-term follow-up. Age alone should not be a deterrent to performing LLIF in the elderly. Additional supplementation may be beneficial in patients with poor bone quality to reduce the risk of graft subsidence.

\section{References}

1. Avila MJ, Walter CM, Baaj AA: Outcomes and complications of minimally invasive surgery of the lumbar spine in the elderly. Cureus 8:e519, 2016

2. Carreon LY, Puno RM, Dimar JR II, Glassman SD, Johnson
JR: Perioperative complications of posterior lumbar decompression and arthrodesis in older adults. J Bone Joint Surg Am 85-A:2089-2092, 2003

3. Cho DC, Sung JK: Palliative surgery for metastatic thoracic and lumbar tumors using posterolateral transpedicular approach with posterior instrumentation. Surg Neurol 71:424433, 2009

4. Deyo RA, Ciol MA, Cherkin DC, Loeser JD, Bigos SJ: Lumbar spinal fusion. A cohort study of complications, reoperations, and resource use in the Medicare population. Spine (Phila Pa 1976) 18:1463-1470, 1993

5. DiPaola CP, Molinari RW: Posterior lumbar interbody fusion. J Am Acad Orthop Surg 16:130-139, 2008

6. Dua K, Kepler CK, Huang RC, Marchenko A: Vertebral body fracture after anterolateral instrumentation and interbody fusion in two osteoporotic patients. Spine J 10:e11-e15, 2010

7. Etzioni DA, Liu JH, Maggard MA, Ko CY: The aging population and its impact on the surgery workforce. Ann Surg 238:170-177, 2003

8. Flynn JC, Price CT: Sexual complications of anterior fusion of the lumbar spine. Spine (Phila Pa 1976) 9:489-492, 1984

9. Fujita T, Kostuik JP, Huckell CB, Sieber AN: Complications of spinal fusion in adult patients more than 60 years of age. Orthop Clin North Am 29:669-678, 1998

10. Grimm BD, Leas DP, Poletti SC, Johnson DR II: Postoperative complications within the first year after extreme lateral interbody fusion: experience of the first 108 patients. Clin Spine Surg 29:E151-E156, 2016

11. Humphreys SC, Hodges SD, Patwardhan AG, Eck JC, Murphy RB, Covington LA: Comparison of posterior and transforaminal approaches to lumbar interbody fusion. Spine (Phila Pa 1976) 26:567-571, 2001

12. Isaacs RE, Podichetty VK, Santiago P, Sandhu FA, Spears J, Kelly K, et al: Minimally invasive microendoscopy-assisted transforaminal lumbar interbody fusion with instrumentation. J Neurosurg Spine 3:98-105, 2005

13. Kanter AS, Gandhoke GS: Lateral lumbar interbody fusion. Neurosurg Focus 35 (2 Suppl):Video 20, 2013

14. Karikari IO, Grossi PM, Nimjee SM, Hardin C, Hodges TR, Hughes BD, et al: Minimally invasive lumbar interbody fusion in patients older than 70 years of age: analysis of peri- and postoperative complications. Neurosurgery 68:897-902, 2011

15. Karikari IO, Nimjee SM, Hardin CA, Hughes BD, Hodges TR, Mehta AI, et al: Extreme lateral interbody fusion approach for isolated thoracic and thoracolumbar spine diseases: initial clinical experience and early outcomes. J Spinal Disord Tech 24:368-375, 2011

16. Kimura I, Shingu H, Murata M, Hashiguchi H: Lumbar posterolateral fusion alone or with transpedicular instrumentation in L4-L5 degenerative spondylolisthesis. J Spinal Disord 14:301-310, 2001

17. Knight RQ, Schwaegler P, Hanscom D, Roh J: Direct lateral lumbar interbody fusion for degenerative conditions: early complication profile. J Spinal Disord Tech 22:34-37, 2009

18. Le TV, Baaj AA, Dakwar E, Burkett CJ, Murray G, Smith DA, et al: Subsidence of polyetheretherketone intervertebral cages in minimally invasive lateral retroperitoneal transpsoas lumbar interbody fusion. Spine (Phila Pa 1976) 37:1268-1273, 2012

19. Malham GM, Ellis NJ, Parker RM, Blecher CM, White R, Goss B, et al: Maintenance of segmental lordosis and disk height in stand-alone and instrumented extreme lateral interbody fusion (XLIF). Clin Spine Surg 30:E90-E98, 2017

20. McAfee PC: Vascular injury during anterior lumbar spine surgery. Spine J 5:118-119, 2005

21. Nikhil N J, Lim JW, Yeo W, Yue WM: Elderly patients achieving clinical and radiological outcomes comparable 
with those of younger patients following minimally invasive transforaminal lumbar interbody fusion. Asian Spine J 11:230-242, 2017

22. Obenchain TG: Laparoscopic lumbar discectomy: case report. J Laparoendosc Surg 1:145-149, 1991

23. Ozgur BM, Aryan HE, Pimenta L, Taylor WR: Extreme lateral interbody fusion (XLIF): a novel surgical technique for anterior lumbar interbody fusion. Spine J 6:435-443, 2006

24. Phan K, Ramachandran V, Tran T, Phan S, Rao PJ, Mobbs RJ: Impact of elderly age on complications and clinical outcomes following anterior lumbar interbody fusion surgery. World Neurosurg 105:503-509, 2017

25. Pradhan BB, Nassar JA, Delamarter RB, Wang JC: Singlelevel lumbar spine fusion: a comparison of anterior and posterior approaches. J Spinal Disord Tech 15:355-361, 2002

26. Raffo CS, Lauerman WC: Predicting morbidity and mortality of lumbar spine arthrodesis in patients in their ninth decade. Spine (Phila Pa 1976) 31:99-103, 2006

27. Rodgers WB, Gerber EJ, Rodgers JA: Lumbar fusion in octogenarians: the promise of minimally invasive surgery. Spine (Phila Pa 1976) 35 (26 Suppl):S355-S360, 2010

28. Schiffman M, Brau SA, Henderson R, Gimmestad G: Bilateral implantation of low-profile interbody fusion cages: subsidence, lordosis, and fusion analysis. Spine J 3:377-387, 2003

29. Stewart Williams J, Ng N, Peltzer K, Yawson A, Biritwum $\mathrm{R}$, Maximova T, et al: Risk factors and disability associated with low back pain in older adults in low- and middleincome countries. Results from the WHO Study on Global AGEing and Adult Health (SAGE). PLoS One 10:e0127880, 2015

30. Tempel ZJ, Gandhoke GS, Okonkwo DO, Kanter AS: Impaired bone mineral density as a predictor of graft subsidence following minimally invasive transpsoas lateral lumbar interbody fusion. Eur Spine J 24 (Suppl 3):414-419, 2015
31. Tempel ZJ, McDowell MM, Panczykowski DM, Gandhoke GS, Hamilton DK, Okonkwo DO, et al: Graft subsidence as a predictor of revision surgery following stand-alone lateral lumbar interbody fusion. J Neurosurg Spine 28:50-56, 2018

\section{Disclosures}

Dr. Okonkwo reports being a consultant for Zimmer Biomet. Dr. Kanter reports being a consultant for NuVasive, and being a patent holder with and receiving royalties from Zimmer Biomet.

\section{Author Contributions}

Conception and design: Kanter, Agarwal, Faramand, Tempel. Acquisition of data: Agarwal, Faramand. Analysis and interpretation of data: Agarwal, Faramand. Drafting the article: Agarwal, Faramand. Critically revising the article: Kanter, Agarwal, Alan, Hamilton, Okonkwo. Reviewed submitted version of manuscript: all authors. Statistical analysis: Faramand. Study supervision: Kanter.

\section{Supplemental Information \\ Previous Presentations}

Data included in this manuscript were presented in oral form at the Society of Lateral Access Surgery Annual Meeting, San Diego, California, May 18-20, 2017; portions were also presented at the 2018 AANS/CNS Section on Disorders of the Spine and Peripheral Nerves meeting, Orlando, Florida, March 14-17, 2018.

\section{Correspondence}

Adam S. Kanter: University of Pittsburgh Medical Center, Pittsburgh, PA.kanteras@upmc.edu. 